\title{
Extremal plurisubharmonic functions
}

\author{
by Urban Cegrell (Umeå) and Johan Thorbiörnson (Sundsvall)
}

Abstract. We study different notions of extremal plurisubharmonic functions.

1. Introduction. There are several different notions of extremal plurisubharmonic functions and the purpose of this note is to study some of them.

DEFInitions. Let $\Omega$ be an open and connected subset of $\mathbb{C}^{n}, n \geq 1$, and denote by $\operatorname{PSH}(\Omega)$ and $\operatorname{PH}(\Omega)$ the plurisubharmonic and pluriharmonic functions, respectively.

Extremal (A). Let $K$ be a subset of $\Omega$. The relative extremal plurisubharmonic function $h_{K}^{*}$ is defined to be the upper regularization of

$$
h_{K}(z)=\sup \{\varphi(z) \in \operatorname{PSH}(\Omega):-1 \leq \varphi \leq 0, \varphi=-1 \text { on } K\}
$$

(cf. Siciak [8]).

Extremal $\left(\mathrm{B}_{1}\right)$. The class of functions $P(\Omega)=\{\varphi \in \operatorname{PSH}(\Omega):-1 \leq \varphi$ $\leq 0\}$ is a convex and compact space with locally convex topology inherited from $L^{1}(\Omega)$. An element $\varphi \in P(\Omega)$ is said to be extremal $\left(\mathrm{B}_{1}\right)$ if

$$
\varphi=\alpha \varphi_{1}+(1-\alpha) \varphi_{2}, \quad \varphi_{1}, \varphi_{2} \in P(\Omega), 0<\alpha<1, \quad \text { implies } \varphi_{1}=\varphi_{2}
$$

(cf. Choquet [3, Vol. II, p. 95]).

Extremal $\left(\mathrm{B}_{2}\right)$. Define $\mathrm{PSH}^{-}(\Omega)=\{\varphi \in \operatorname{PSH}(\Omega): \varphi \leq 0\}$. An element $\varphi \in \mathrm{PSH}^{-}(\Omega)$ is said to be extremal $\left(\mathrm{B}_{2}\right)$ if

$$
\varphi=\varphi_{1}+\varphi_{2}, \quad \varphi_{1}, \varphi_{2} \in \operatorname{PSH}^{-}(\Omega)
$$

implies that there exist non-negative constants $\lambda_{1}$ and $\lambda_{2}$ such that $\varphi_{1}=\lambda_{1} \varphi$ and $\varphi_{2}=\lambda_{2} \varphi$.

1991 Mathematics Subject Classification: 31C05, 31C10, 32F05.

Key words and phrases: extremal plurisubharmonic function, pluricomplex Green function. 
Extremal (C). The quotient $\operatorname{space} \operatorname{PSH}(\Omega) / \operatorname{PH}(\Omega)$ is Hausdorff and a convex cone. An element $\varphi \in \operatorname{PSH}(\Omega) / \operatorname{PH}(\Omega)$ is called extremal (C) if

$$
\varphi=\varphi_{1}+\varphi_{2}, \quad \varphi_{1}, \varphi_{2} \in \operatorname{PSH}(\Omega) / \operatorname{PH}(\Omega),
$$

implies that there exist non-negative constants $\lambda_{1}$ and $\lambda_{2}$ such that $\varphi_{1}=\lambda_{1} \varphi$ and $\varphi_{2}=\lambda_{2} \varphi$ (in $\operatorname{PSH}(\Omega) / \operatorname{PH}(\Omega)$ ) (cf. Lelong [5] and Demailly [4]).

2. The case $n=1$. Let here $\Omega=D$ be the open unit disc in the complex plane and $\varphi \in \mathrm{SH}(D)$ with a harmonic majorant. Then, by the Riesz representation formula,

$$
\varphi(z)=\int \log \left|\frac{z-\xi}{1-z \bar{\xi}}\right| \Delta \varphi(\xi)+\int P(z, \xi) d \mu(\xi),
$$

where $P$ is the Poisson kernel. So that if $\varphi \leq 0$ and $\varphi$ is extremal $\left(\mathrm{B}_{2}\right)$ then either $\Delta \varphi \equiv 0$ or $\mu=0$. Therefore $\Delta \varphi=k \delta_{z_{0}}$ for some $z_{0} \in \Omega$ so

$$
\varphi(z)=k \log \left|\frac{z-z_{0}}{1-z \bar{z}_{0}}\right|
$$

or $\varphi(z)=k P\left(z, \xi_{0}\right)$ for some $\xi_{0} \in \partial \Omega$, with $k$ a constant.

Also, if $\varphi \in \mathrm{SH}(D)$ is extremal $(\mathrm{C})$ then $\varphi(z)=k \log \left|z-z_{0}\right|$ modulo a harmonic function. Therefore, there are no functions bounded below and extremal (C).

\section{Relations}

LEMmA 1. If $0,-1 \not \equiv \varphi \in P(\Omega)$ is extremal $\left(\mathrm{B}_{1}\right)$, then $\inf _{z \in \Omega}=-1$ and $\sup _{z \in \Omega} \varphi(z)=0$.

Proof. If $\beta=\inf _{z \in \Omega} \varphi(z)>-1$, then $\varphi(z) / \gamma \in P(\Omega)$, where $\gamma=$ $\max (1 / 2,-\beta)<1$. Choose $k$ so that $1 / \gamma+k=2$. Since $1<1 / \gamma \leq 2$ it follows that $0 \leq k \leq 1$ so $k \varphi(z) \in P(\Omega)$ and

$$
\varphi(z)=\frac{1}{2}\left(\frac{\varphi(z)}{\gamma}+k \varphi(z)\right)
$$

so $\varphi$ is not extremal $\left(\mathrm{B}_{1}\right)$. If $\sup _{z \in \Omega} \varphi(z)=\delta<0$ then

$$
\varphi(z)=\varphi(z)+\delta-\delta=\left(\frac{\varphi(z)+\delta}{1-\delta}\right)(1-\delta)+\delta \cdot(-1)
$$

so $\varphi$ is not extremal.

Proposition 1. If $K$ is a relatively compact subset of $\Omega$ then $h_{K}^{*}$ is extremal $\left(\mathrm{B}_{1}\right)$.

Proof. If $K$ is pluripolar, then $h_{K}^{*} \equiv 0$; we can assume that $K$ is not pluripolar. If $h_{K}^{*}=\alpha u_{1}+(1-\alpha) u_{2}$, where $u_{1}, u_{2} \in P(\Omega)$, then $u_{1}=u_{2}=$ -1 on $\left\{z \in \Omega: h_{K}^{*}=-1\right\}$ so it follows from the definition of $h_{K}$ (since 
$h_{K}=h_{K}^{*}$ outside a pluripolar set) that $u_{1} \leq h_{K}^{*}$ and $u_{2} \leq h_{K}^{*}$. Therefore $u_{1}=u_{2}=h_{K}^{*}$.

Corollary. There exist discontinuous functions extremal $\left(\mathrm{B}_{1}\right)$.

Proposition 2. If $\varphi \in \operatorname{PSH}^{-}(\Omega), \lim _{z \rightarrow \xi} \varphi(z)=0$ for all $\xi \in \partial \Omega$ and if $\varphi$ is extremal $(\mathrm{C})$, then $\varphi$ is extremal $\left(\mathrm{B}_{2}\right)$. If $\varphi \in P(\Omega)$ with $\varphi\left(\xi_{0}\right)=-1$ for some $\xi_{0} \in \Omega$ and if $\varphi$ is extremal $\left(\mathrm{B}_{2}\right)$ then $\varphi$ is extremal $\left(\mathrm{B}_{1}\right)$.

Proof. Suppose $\varphi \in \operatorname{PSH}^{-}(\Omega)$ and that $\varphi$ is extremal (C). If $\varphi=$ $\varphi_{1}+\varphi_{2}$, where $\varphi_{1}, \varphi_{2} \in \operatorname{PSH}^{-}(\Omega)$, then

$$
\begin{array}{ll}
\varphi_{1}+h_{1}=\lambda_{1} \varphi, & \lambda_{1} \geq 0, h_{1} \in \mathrm{PH}, \\
\varphi_{2}+h_{2}=\lambda_{2} \varphi, & \lambda_{2} \geq 0, h_{2} \in \mathrm{PH} .
\end{array}
$$

Since $\varphi=\varphi_{1}+\varphi_{2}$ and $\varphi_{1}, \varphi_{2} \leq 0$, also $\varphi_{1}(z), \varphi_{2}(z) \rightarrow 0$ as $z \rightarrow \xi, \xi \in \partial \Omega$. Hence $h_{1}(z), h_{2}(z) \rightarrow 0$ as $z \rightarrow \xi, \xi \in \partial \Omega$, since $h_{1}=\lambda_{1} \varphi-\varphi_{1}$ and $h_{2}=\lambda_{2} \varphi-\varphi_{2}$. Therefore, being harmonic, $h_{1}$ and $h_{2}$ vanish identically on $\Omega$.

Let now $\varphi \in P(\Omega)$ be extremal $\left(\mathrm{B}_{2}\right)$ and assume $\varphi\left(\xi_{0}\right)=-1$ for some $\xi_{0} \in \Omega$. If

$$
\varphi=\alpha \varphi_{1}+(1-\alpha) \varphi_{2}, \quad 0<\alpha<1, \varphi_{1}, \varphi_{2} \in P(\Omega),
$$

then

$$
\begin{aligned}
\alpha \varphi_{1} & =\lambda_{1} \varphi, & & \lambda_{1} \geq 0, \\
(1-\alpha) \varphi_{2} & =\lambda_{2} \varphi, & & \lambda_{2} \geq 0 .
\end{aligned}
$$

At $\xi_{0}$, we have $-1=\varphi\left(\xi_{0}\right)=\varphi_{1}\left(\xi_{0}\right)=\varphi_{2}\left(\xi_{0}\right)$ so $\alpha=\lambda_{1}$ and $1-\alpha=\lambda_{2}$, which proves that $\varphi$ is extremal $\left(\mathrm{B}_{1}\right)$.

Proposition 3. Suppose $0 \geq \varphi \in \operatorname{PSH}(\Omega \times \Omega)$ and that $\varphi$ is separately extremal $\left(\mathrm{B}_{2}\right)$. Then $\varphi$ is extremal $\left(\mathrm{B}_{2}\right)$ on $\Omega \times \Omega$.

Proof. Suppose $\varphi=\varphi_{1}+\varphi_{2}$, where $0 \geq \varphi_{1}, \varphi_{2} \in \operatorname{PSH}(\Omega \times \Omega)$. Then

$$
\begin{aligned}
& \varphi_{1}(x, y)=\lambda_{1}(y) \varphi(x, y)=\beta_{1}(x) \varphi(x, y), \\
& \varphi_{2}(x, y)=\lambda_{2}(y) \varphi(x, y)=\beta_{2}(x) \varphi(x, y),
\end{aligned}
$$

where $\lambda_{1}, \lambda_{2}, \beta_{1}, \beta_{2} \geq 0$ and $\lambda_{1}+\lambda_{2}=1=\beta_{1}+\beta_{2}$ since $\varphi$ is separately extremal $\left(\mathrm{B}_{2}\right)$. Therefore $\lambda_{1} \equiv \beta_{1} \equiv$ const and $\lambda_{2} \equiv \beta_{2} \equiv$ const, which proves that $\varphi$ is extremal $\left(\mathrm{B}_{2}\right)$.

Lemma 2. Suppose $0 \geq u \in \operatorname{PSH}(G)$, where $G$ is a ball in $\mathbb{C}^{n}$ with center at 0 . Then

$$
\lim _{r \searrow 0} \sup _{\substack{\xi \in \mathbb{C} \\|\xi|=r}} \frac{u(\xi \nu)}{-\log r}=\mathrm{const}
$$

for all $\nu \in G$ outside a pluripolar set. 
Proof. We may assume that $G=B$, the unit ball. Suppose $0 \geq u \in$ $\operatorname{PSH}(B)$. For $0<r<1$ and $\nu \in B$ we define

$$
\Psi_{r}(\nu)=\sup _{\substack{\xi \in \mathbb{C} \\|\xi|=r}} \frac{u(\xi \nu)}{-\log r} \leq 0 .
$$

Then $\Psi_{r}(\nu) \in \operatorname{PSH}(B)$ and is decreasing in $r$. For let $0<r_{1}<r_{2}<1$. If $z \in \mathbb{C}$ with $|z|=r_{1}$, then $u(z \nu) \leq \Psi_{r_{1}}(\nu) \cdot(-\log |z|)$, which also holds for $|z|=1$. Therefore $u(z \nu) \leq \Psi_{r_{1}}(\nu) \cdot(-\log |z|)$ for $r_{1} \leq|z| \leq 1$. In particular,

$$
\frac{u(z \nu)}{-\log |z|} \leq \Psi_{r_{1}}(\nu) \quad \text { for }|z|=r_{2}
$$

and so $\Psi_{r_{2}}(\nu) \leq \Psi_{r_{1}}(\nu)$.

Hence $\Psi^{*}(\nu)=\left(\lim _{r}{ }_{0} \Psi_{r}(\nu)\right)^{*} \in \operatorname{PSH}\left(\mathbb{C}^{n}\right)$ and since $\Psi^{*}$ is negative it is constant. Since $\Psi=\Psi^{*}$ outside a pluripolar set (cf. [1]), the lemma follows.

Rem ark 1 . The constant $-\Psi^{*}$ can be shown to be equal to the Lelong number of $u$ at zero. We do not need to use that in this paper.

\section{Examples}

Example 1 . Denote by $D$ the unit disc in $\mathbb{C}$. Then

$$
h_{\left\{\left|z_{1}\right|<e^{-1}\right\} \times\left\{\left|z_{2}\right|<e^{-1}\right\}}^{*}(z)=\max \left(\log \left|z_{1}\right|, \log \left|z_{2}\right|,-1\right)
$$

so $\max \left(\log \left|z_{1}\right|, \log \left|z_{2}\right|,-1\right), z=\left(z_{1}, z_{2}\right) \in D \times D$, is extremal (A) and thus by Proposition 1 also extremal $\left(\mathrm{B}_{1}\right)$.

Example 2. Denote by $B$ the unit ball in $\mathbb{C}^{n}$. Then

$$
h_{\left\{z \in \mathbb{C}^{n}:|z|<e^{-1}\right\}}^{*}(z)=\max (\log |z|,-1)
$$

is extremal $(\mathrm{A})$ and therefore extremal $\left(\mathrm{B}_{1}\right)$.

ExAmple 3. If $\alpha>0$ then $\varphi(z)=|z|^{\alpha}-1$ is not extremal $\left(\mathrm{B}_{1}\right)$ on $D$. For let

$$
\varphi_{1}(z)=\frac{4}{3}\left(|z|^{\alpha}-\frac{|z|^{2 \alpha}}{4}-\frac{3}{4}\right), \quad \varphi_{2}(z)=\frac{2}{3}\left(|z|^{\alpha}+\frac{|z|^{2 \alpha}}{2}-\frac{3}{2}\right) .
$$

Then $\varphi=\left(\varphi_{1}+\varphi_{2}\right) / 2$, and since $\Delta \varphi_{1}=\alpha^{2}\left(|z|^{\alpha-2}-|z|^{2 \alpha-2}\right) \geq 0$ for $0 \leq|z|<1, \varphi_{1}$ is subharmonic on $D$ and therefore $\varphi_{1}, \varphi_{2} \in P(D)$.

EXAMPLE 4. $\psi\left(z_{1}, z_{2}\right)=\max \left(\log \left|z_{1}\right|, \log \left|z_{2}\right|\right)$ is not extremal (C). Since

$$
\frac{1}{2 \pi} \int_{0}^{2 \pi} \log \left|\omega-e^{i \theta}\right| d \theta=\max (\log |\omega|, 0), \quad \omega \in \mathbb{C},
$$


it follows that

$$
\psi\left(z_{1}, z_{2}\right)=\max \left(\log \left|z_{1}\right|, \log \left|z_{2}\right|\right)=\frac{1}{2 \pi} \int_{0}^{2 \pi} \log \left|z_{1}+z_{2} e^{i \theta}\right| d \theta .
$$

Thus

$$
\psi\left(z_{1}, z_{2}\right)=\frac{1}{2 \pi} \int_{0}^{\pi} \log \left|z_{1}+z_{2} e^{i \theta}\right| d \theta+\frac{1}{2 \pi} \int_{\pi}^{2 \pi} \log \left|z_{1}+z_{2} e^{i \theta}\right| d \theta
$$

so $\psi$ is not extremal $(\mathrm{C})$.

However, $\psi$ is extremal $\left(\mathrm{B}_{2}\right)$ in $D \times D$. For suppose $\psi=\varphi_{1}+\varphi_{2}$, where $\varphi_{1}, \varphi_{2} \in \mathrm{PSH}^{-}(D \times D)$. If $z=\left(z_{1}, z_{2}\right)$, then $\psi(\lambda z), \lambda \in \mathbb{C}$, is extremal as a function of $\lambda$, so

$$
\begin{array}{ll}
\varphi_{1}(\lambda z)=\alpha_{2} \psi(\lambda z)=\alpha_{2}(\log |\lambda|+\psi(z)), & \alpha_{2} \geq 0, \\
\varphi_{2}(\lambda z)=\beta_{2} \psi(\lambda z)=\beta_{2}(\log |\lambda|+\psi(z)), & \beta_{2} \geq 0 .
\end{array}
$$

By Lemma $2, \alpha_{2}=$ const $=\alpha$ a.e., $\beta_{2}=$ const $=\beta$ a.e. Therefore $\varphi_{1}(z)=$ $\alpha \varphi(z)$ and $\varphi_{2}(z)=\beta \varphi(z)$, which means that $\psi$ is extremal $\left(\mathrm{B}_{2}\right)$.

Remark 2. Example 4 answers a question by El Mir [6].

ExAMPLE 5 (Poletsky [7]). The function $\log |z|$ is extremal $\left(\mathrm{B}_{2}\right)$ in the unit ball of $\mathbb{C}^{n}$. This can be shown exactly as in Example 4, using Lemma 2.

5. The pluricomplex Green function. The functions $\max \left(\log \left|z_{1}\right|\right.$, $\left.\log \left|z_{2}\right|\right)$ and $\log |z|$ are pluricomplex Green functions with pole at zero for the domains $D \times D$ and $B$ respectively. Example 4 shows that the pluricomplex Green function is not extremal (C) in the bidisc. Using a suitable holomorphic transformation, Examples 4 and 5 show that the pluricomplex Green function for the bidisc and the ball is extremal $\left(\mathrm{B}_{2}\right)$. This is a special example of the following theorem.

TheOREM. Let $\Omega$ be a domain in $\mathbb{C}^{n}$ and let $G_{\Omega}(x, y)$ be the pluricomplex Green function for $\Omega \times \Omega$. Then $G_{\Omega}\left(x, y_{0}\right)$ is extremal $\left(\mathrm{B}_{2}\right)$ for every $y_{0} \in \Omega$.

Proof. We can assume that $y_{0}=0$. Suppose $G_{\Omega}=\varphi_{1}+\varphi_{2}$, where $\varphi_{1}, \varphi_{2} \in \mathrm{PSH}^{-}(\Omega)$. Using Lemma 2 , we find three constants

$$
\begin{gathered}
\alpha=\left(\lim _{r \searrow 0} \sup _{\xi} \frac{G_{\Omega}(\xi \nu)}{-\log |\xi|}\right)^{*}, \\
\alpha_{1}=\left(\lim _{r \searrow 0} \sup _{\substack{\xi|=r\\
| \xi \mid=r}} \frac{\varphi_{1}(\xi \nu)}{-\log |\xi|}\right)^{*} \text { and } \alpha_{2}=\left(\lim _{r \searrow 0} \sup _{\xi \xi} \frac{\varphi_{2}(\xi \nu)}{-\log |\xi|}\right)^{*} .
\end{gathered}
$$

Since $G_{\Omega}(x, 0)-\log |x|$ is bounded near $x=0$, we have $\alpha=-1,0 \geq \alpha_{1}, \alpha_{2} \geq$ -1 and $\alpha_{1}+\alpha_{2} \geq-1$. 
Let $V$ be a ball in $\Omega$ centered at zero. We consider only the points $\nu \in V$ where the regularization $*$ is not necessary. This is enough, since the complement of this set is pluripolar. Without loss of generality, we can also assume that $V=B$, the unit ball.

Note that

$$
\frac{\varphi_{1}(\xi \nu)}{-\log |\xi|} \leq \alpha_{1}, \quad \frac{\varphi_{2}(\xi \nu)}{-\log |\xi|} \leq \alpha_{2}, \quad|\xi| \leq 1
$$

Hence $\varphi_{1}(\xi \nu) \leq-\alpha_{1} \log |\xi|$ and $\varphi_{2}(\xi \nu) \leq-\alpha_{2} \log |\xi|$ so $\varepsilon_{1}(\xi)=\varphi_{1}(\xi \nu)+$ $\alpha_{1} \log |\xi|$ and $\varepsilon_{2}(\xi)=\varphi_{2}(\xi \nu)+\alpha_{2} \log |\xi|$ both extend to subharmonic functions on the unit disc. Furthermore, since

$$
\sup _{\substack{r \geq 0 \\ \xi \in \mathbb{C} \\|\xi|=r}} \frac{\varepsilon_{1}(\xi)}{-\log |r|}=\sup _{\substack{r \geq 0 \\ \xi \in \mathbb{C} \\|\xi|=r}} \frac{\varepsilon_{2}(\xi)}{-\log |r|}=0,
$$

we have $\Delta \varepsilon_{1}\{0\}=\Delta \varepsilon_{2}\{0\}=0$. Also, since

$$
\begin{aligned}
G_{\Omega}(\xi \nu, 0) & =\varphi_{1}(\xi \nu)+\varphi_{2}(\xi \nu)=\varepsilon_{1}(\xi)+\varepsilon_{2}(\xi)-\alpha_{1} \log |\xi|-\alpha_{2} \log |\xi| \\
& =\log |\xi|+\varepsilon_{1}(\xi)+\varepsilon_{2}(\xi)-\left(1+\alpha_{1}+\alpha_{2}\right) \log |\xi|
\end{aligned}
$$

and since $\xi \mapsto G_{\Omega}(\xi \nu, 0)-\log |\xi|$ is subharmonic on the unit disc, it follows that $-\left(1+\alpha_{1}+\alpha_{2}\right) \geq 0$. Thus $\alpha_{1}+\alpha_{2} \leq-1$ and as we already know that $\alpha_{1}+\alpha_{2} \geq-1$, we conclude that $\alpha_{1}+\alpha_{2}=-1$.

Now

$$
\frac{\varphi_{1}(\xi \nu)}{-\alpha_{1}} \leq \log |\xi|, \quad \frac{\varphi_{2}(\xi \nu)}{-\alpha_{2}} \leq \log |\xi|, \quad|\xi| \leq 1
$$

so

$$
\frac{\varphi_{1}(z)}{-\alpha_{1}} \leq G_{\Omega}(z, 0), \quad \frac{\varphi_{2}(z)}{-\alpha_{2}} \leq G_{\Omega}(z, 0), \quad z \in B,
$$

by the definition of $G_{\Omega}$. Since $\varphi_{1}+\varphi_{2} \equiv G_{\Omega}$, it follows that $\varphi_{1} /\left(-\alpha_{1}\right)=$ $\varphi_{2} /\left(-\alpha_{2}\right)=G_{\Omega}$.

R e mark 3. From the proof of Proposition 3, it follows that if $\Omega$ is such that $G_{\Omega}$ is symmetric (for example, if $\Omega$ is convex), then $G_{\Omega}$ is "extremal $\left(\mathrm{B}_{2}\right)$ " among the negative separately plurisubharmonic functions.

R e mark 4. In the unit disc, every bounded subharmonic function is an (infinite) sum of subharmonic and continuous functions. We do not know if this is true in the bidisc (cf. [2]), but this problem is one of the motivations for us to study extremal plurisubharmonic functions. 


\section{References}

[1] E. Bedford and B. A. Taylor, A new capacity for plurisubharmonic functions, Acta Math. 149 (1982), 1-40.

[2] U. Cegrell, Sums of continuous plurisubharmonic functions and the Monge-Ampère operator in $\mathbb{C}^{n}$, Math. Z. 193 (1986), 373-380.

[3] G. Choquet, Lectures on Analysis, Benjamin, New York, 1969.

[4] J.-P. Demailly, Courants positifs extrémaux et conjecture de Hodge, Invent. Math. 69 (1982), 347-374.

[5] P. Lelong, Eléments extrémaux dans le cône des courants positifs fermés de type $(1,1)$ et fonctions plurisousharmoniques extrémales, C. R. Acad. Sci. Paris Sér. A 273 (1971), 665-667.

[6] H. El Mir, personal communication.

[7] E. A. Poletsky, personal communication.

[8] J. Siciak, Extremal Plurisubharmonic Functions and Capacities in $\mathbb{C}^{n}$, Sophia Kokyuroku in Math. 14, Sophia University, Tokyo, 1982.

DEPARTMENT OF MATHEMATICS

UNIVERSITY OF UMEÅ

S-901 87 UMEA, SWEDEN

E-mail: URBAN.CEGRELL@MATHDEPT.UMU.SE
DEPARTMENT OF MATHEMATICS MID SWEDEN UNIVERSITY S-851 70 SUNDSVALL, SWEDEN E-mail: JOHANT@NTS.MH.SE

Reçu par la Rédaction le 16.9.1994

Révisé le 15.1.1995 